\title{
Nectines et nectines-like
}

\section{Marqueurs, acteurs et cibles de l'oncogenèse}

$>$ Les nectines et nectines-like (necl) constituent une famille de molécules d'adhérence composée de 9 membres appartenant à la superfamille des Gaëlle Fournier, Sarah Garrido-Urbani, Nicolas Reymond, Marc Lopez immunoglobulines. Elles jouent un rôle majeur dans différents processus biologiques comme la polarité cellulaire, la prolifération, la différenciation et la migration cellulaire. Leurs actions sont réparties dans divers compartiments comme les épithéliums, les endothéliums, les systèmes immunitaire et nerveux. Elles sont aussi impliquées dans différents processus pathologiques chez l'homme. Elles jouent le rôle de récepteurs de virus (virus de la poliomyélite, virus herpes simplex), sont impliquées dans des malformations orofaciales (CLPED1, cleft lip/palate-ectodermal dysplasia syndrome) et plus récemment elles ont été décrites comme marqueurs, acteurs et cibles thérapeutiques potentielles dans le cancer. Parmi elles, necl-5, nectine-2 et nectine- 4 sont surexprimées dans les tumeurs et sont associées à un mauvais pronostic. D'autres, comme necl-1, necl-2, et necl-4 sont des gènes suppresseurs de tumeurs dont l'expression est perdue dans les cancers. Le rôle de ces molécules dans le cancer et leur potentielle utilisation thérapeutique sont discutés dans cette revue. <

\section{Le cancer : un problème de santé publique}

Le cancer est un problème majeur de santé publique à l'échelle mondiale. Même si de réels progrès ont été réalisés ces dix dernières années en matière de prévention, dépistage, diagnostic précoce, traitements et prise en charge, ces avancées restent encore insuffisantes. Ceci est principalement lié au fait que pour un même type de cancer, il existe une hétérogénéité histologique, clinique et moléculaire marquée. Le traitement individualisé des cancers prenant en compte cette hétérogénéité pourrait améliorer leur prise en charge. Les avancées thérapeutiques passent notamment par l'identification de nouveaux

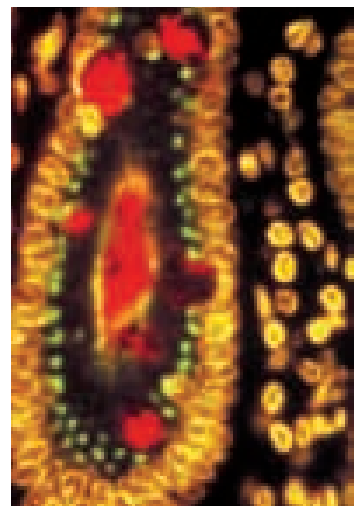

marqueurs exprimés ou surexprimés par certaines tumeurs (antigènes associés aux tumeurs). G. Fournier, M. Lopez : Inserm, UMR 891 ; Centre de recherche en cancérologie de Marseille, F-13009, France ;

Université de la Méditerranée, Marseille, F-13007, France. S. Garrido-Urbani : Department of Pathology and Immunology, Centre médical universitaire, 1211 Genève 4, Suisse. N. Reymond: Randall Division of Cell and Molecular Biophysics, King's College London, London SEl IUL, Royaume-Uni. gaelle.fournier@inserm.fr marc.lopez@inserm.fr Ces marqueurs de diagnostic précoce et/ou de suivi thérapeutique peuvent aussi représenter des cibles thérapeutiques s'ils contribuent au développement tumoral. Ces cibles sont fréquemment des protéines de surface (récepteur à activité tyrosine kinase, molécule d'adhérence) impliquées dans la progression tumorale (prolifération, adhérence, migration, transmigration) et sont éligibles aux approches d'immunothérapie. Àce jour, les thérapies ciblées utilisent principalement: (1) des inhibiteurs de l'activité catalytique de kinases participant au développement tumoral, (2) des anticorps humanisés dirigés contre des antigènes de surface tumoraux (biothérapie ou immunothérapie $)^{1}$, (3) la vaccination antitumorale thérapeutique ou prophylactique post-traitement de consolidation (immunothérapie active).

Dans cette revue, nous décrirons la famille de molécules d'adhérence appelées nectines et nectines-like (necl) dont les membres sont des cibles naturelles des lymphocytes cytolytiques et dont l'expression est régulée spécifiquement dans les cancers. Une expression augmentée des nectines et des nectines-like facilite

${ }^{1}$ Voir le numéro thématique de décembre 2009 de Médecine/Sciences consacré aux anticorps monoclonaux en thérapeutique. 

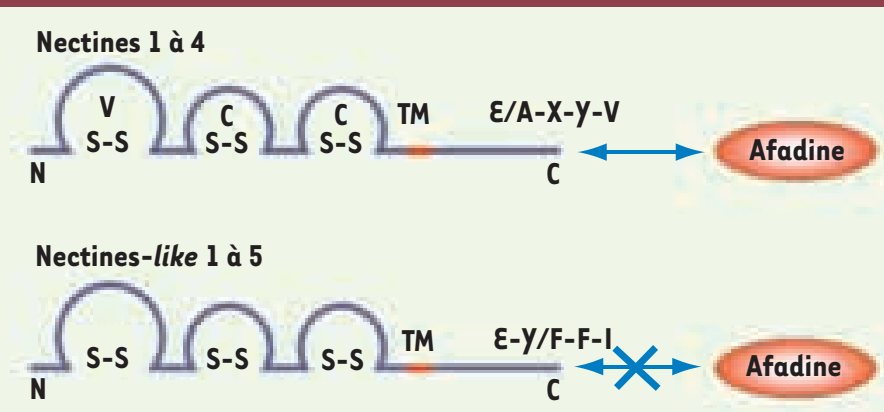

le développement de tumeurs via la prolifération et la migration. Par ailleurs, la perte d'expression est associée à la perte de la fonction suppresseur de tumeurs de la protéine. Certains de ces membres constituent donc une nouvelle classe d'antigènes tumoraux potentiellement éligibles comme marqueurs, acteurs et cibles thérapeutiques.

\section{Les protéines nectines et nectines-like : molécules d'adhérence des jonctions intercellulaires}

Les nectines et les nectines-like sont des molécules d'adhérence appartenant à la superfamille des immunoglobulines (SFIg) qui jouent un rôle essentiel dans la formation des jonctions intercellulaires, la polarité, la prolifération, la différenciation, la migration et la survie cellulaires.

La famille des nectines et des nectines-like comprend quatre nectines (1 à 4) [1-4] et cinq nectines-like (1 à 5) [5-8]. Ces protéines sont composées d'une région extracellulaire constituée de trois domaines apparentés aux domaines des immunoglobulines, d'une région transmembranaire et d'une région cytoplasmique. De nombreuses protéines sont capables d'interagir de manière directe ou indirecte avec les nectines et les nectines-like via leur région cytoplasmique. Parmi elles, l'afadine est une protéine plateforme qui relie les nectines au cytosquelette d'actine [9] mais n'interagit pas avec les nectines-like. Cette particularité permet de distinguer les nectines des nectines-like (Figure 1).

Les molécules nectines et nectines-like forment des cis-dimères à la surface d'une cellule via leur domaine extracellulaire. Ces cis-dimères forment des interactions en trans, homophiliques (entre deux mêmes molécules) et hétérophiliques (entre deux molécules différentes) entre nectines $[2,4,9,10]$ et nectines-like [11] exprimées à la surface de cellules adjacentes. Des membres de la famille des nectines et nectineslike sont aussi capables de former des interactions hétérophiliques en trans avec d'autres partenaires appartenant à la SFIg tels que DNAMI (DNAX accessory molecule-1 ou CD226) [12], tactile (CD96) [13], CRTAM (Class I-restricted T cell-associated molecule) [14] et WUCAM (Washington University cell adhesion molecule) [15]. Ainsi, un réseau complexe de 17 trans-interactions hétérophiliques différentes avec les protéines nectines et nectines-like est actuellement décrit, certaines nectines étant associées à une fonction biologique (Figure 2).

Les nectines et les nectines-like sont exprimées dans différents types cellulaires comme les cellules épithéliales, endothéliales, fibroblastiques, hématopoïétiques, nerveuses et germinales. Dans l'épithélium, la localisation des nectines est restreinte aux jonctions adhérentes $(\mathrm{JA})$ et les
Figure 1. Structure des nectines et des nectines-like. Cette famille de molécules d'adhérence appartient à la SFIg et ces protéines présentent trois domaines de type $\lg$ V, C, C. Les domaines IgV participent aux interactions hétérotypiques et sont les domaines d'interactions avec les virus. Les domaines $\lg C$ participent aussi aux interactions homophiliques et à la cis-dimérisation. Le motif d'interaction des nectines avec le domaine PDZ de l'afadine est $\varepsilon / A-X-Y-V$. Pour la nectine-4, le motif est G-H-L-V.

nectines sont impliquées dans la formation des JA et des jonctions serrées (JS) des cellules épithéliales et des fibroblastes. Cette localisation n'est pas exclusive, les nectines participant à de nombreuses autres interactions hétérotypiques (entre deux types cellulaires différents) (Figure 2).

Les nectines initient les premiers contacts entre cellules adjacentes et contribuent au recrutement des cadhérines et à la formation et la stabilisation des JA. Elles interviennent dans la régulation de la cadhérine- $\varepsilon$ avec laquelle elles interagissent grâce aux molécules respectivement associées à leur domaine cytoplasmique. II s'agit principalement de l'afadine/AF6 et de l' $\alpha$-caténine qui peuvent interagir directement ou indirectement par le biais de protéines adaptatrices: ponsine - vinculine et $\alpha$-actinine-ADIP (Afadin DIL domain-interacting protein) /LM07 (LIM domain only 7) [16] (Figure 3A). Les nectines interagissent aussi avec la protéine de polarité PAR3 (partitioning defective-3) [16].

Les nectines, comme les cadhérines, ont la capacité d'activer des molécules impliquées dans des voies de signalisation comme les petites Rho GTPases, RACl et CDC42 par l'intermédiaire de la tyrosine kinase SRC [16] (Figure 3A). L'activation de RACl et CDC42 favorise la motilité cellulaire et contribue à la formation de structures cellulaires impliquées dans le mouvement des cellules que sont les lamellipodes et des filopodes. Lorsque RACl et CDC42 sont activées, elles permettent le recrutement des molécules liées aux filaments d'actine, comme IQGAPI (IO motif containing GTPase activating protein 1) permettant de stabiliser les JA [16].

Au niveau des JA, les complexes nectines-afadine et cadhérines-caténines recrutent de nombreuses protéines et activent des voies de signalisation afin de réorganiser le cytosquelette d'actine pour former des jonctions stables entre cellules adjacentes [16].

Certaines nectines-like ont des expressions spécifiques au tissu nerveux et d'autres sont retrouvées dans la région basolatérale des épithéliums. Les nectines-like interagissent avec des protéines plateforme telles que les MAGUK (membrane associated guanylate kinase) 
(PALS2, DLG3, CASK) et des membres de la famille de la protéine de la bande 4.1 (DALl) [17]. Necl-5 interagit avec la protéine TCTEX-1, un composant du moteur moléculaire dynéine [18].

\section{Les nectines et les nectines-like : implication en cancérologie}

Les nectines et nectines-like sont impliquées dans différents processus pathologiques. Necl-5/PVR est le récepteur du virus de la poliomyélite et nectine- 1 le récepteur majeur des virus herpes simplex de types 1 et $2[8,19]$. Des mutations du gène nectine- 1 sont responsables de malformations orofaciales accompagnées de retard mental [20]. Plus récemment, certains membres de cette famille ont été impliqués dans la physiopathologie des cancers. La surexpression ou la perte d'expression des nectines et des nectines-like par des cellules tumorales suggère que ces molécules peuvent jouer un rôle dans la transformation cellulaire et/ou dans la progression tumorale. Ces molécules pourraient être essentielles pour la survie et le maintien des propriétés tumorales des cellules cancéreuses (Tableau I).

\section{Gènes activateurs de tumeurs}

\section{Nectine-2 et necl-5 : rôle central dans la progression tumorale}

Une caractéristique des molécules nectines et nectines-like est leur surexpression dans des tumeurs d'ori- gine très variée: neuronales, épithéliales et hématopoïétiques. Cette surexpression engendre deux types d'effets : des effets directs sur le comportement des cellules tumorales, stimulant leur progression, et des effets stimulant la réponse immune innée cytolytique, puisque ces molécules sont des cibles des effecteurs de l'immunité innée. Ces deux types d'effets ont été décrits et peuvent paraître contradictoires, mais les observations ont été faites par des laboratoires indépendants et dans des contextes expérimentaux différents, sans que la balance entre les effets pro- et anti-tumoraux des nectines et nectines-like ait été précisément analysée [21].

Nectine-2 et necl-5 sont des ligands de DNAM-1, une molécule exprimée par les cellules de l'immunité natural killer (NK), des sous-populations lymphocytaires, et les monocytes. DNAM-1 est un récepteur activateur des cellules NK indispensable à leur fonction cytotoxique [12] (Figure 3B). Chez l'homme, les récepteurs NK activateurs sont principalement NKp46, NKp30, NKp44 (appelés NCR et exprimés spécifiquement sur les cellules NK) et DNAM- 1 et NKG2D. Les cellules NK expriment des récepteurs qui reconnaissent les molécules HLA de classe I et transduisent des signaux inhibiteurs des fonctions lytiques NK. Ainsi, l'absence ou la diminution de l'expression des molécules HLA de classe I par les cellules tumorales, souvent accompagnée de l'augmentation de l'expression des ligands des récepteurs NK activateurs, contribue à favoriser la lyse des cellules tumorales. DNAM-l contribue à la lyse de tumeurs d'origine hématologique et non hématologique [22]. L'importance de DNAM-1 dans l'immunosurveillance des tumeurs a été prouvée in vivo chez la souris. L'expression ectopique des nectine- 2 et necl- 5 est suffisante pour empêcher le développement tumoral de cellules tumorales inoculées dans la souris [23]. De plus, le développement de tumeurs est accéléré dans les souris déficientes pour DNAM-1 [24]. L'interaction entre DNAM-1 et ses ligands, nectine-2 et necl-5, joue un rôle important dans le rejet

\begin{tabular}{|c|c|c|}
\hline Nomenclature & Autres nomenclatures & Pathologies et phénotypes associés \\
\hline Nectine-1 & PRR1, HveC, CD111 & $\begin{array}{l}\text { Récepteur d'entrée des virus herpes simplex type } 1 \text { et } 2 \\
\text { Mutée dans le syndrome de CLPED } \\
\text { KO : microphtalmie, anomalies de la peau et de l'hippocampe }\end{array}$ \\
\hline Nectine-2 & PRR2, HveB, CD112 & $\begin{array}{l}\text { Récepteur d'entrée du virus herpes simplex type } 2 \\
\text { KO : mâle stérile }\end{array}$ \\
\hline Nectine-3 & PRR3, CD113 & KO : microphtalmie, anomalie de l'hippocampe, mâle stérile \\
\hline Nectine- 4 & PRR4 & Surexprimée dans le cancer du sein et du poumon \\
\hline Necl-1 & TSLLl, SynCAM3 & Suppresseur de tumeur dans les gliomes et le cancer du poumon \\
\hline $\mathrm{Necl}-2$ & $\begin{array}{l}\text { RA175, IGSF4, SgIGSF, TSLCl, } \\
\text { SynCAMl }\end{array}$ & $\begin{array}{l}\text { Suppresseur de tumeur dans le cancer du poumon } \\
\text { KO : mâle stérile }\end{array}$ \\
\hline $\mathrm{Necl}-3$ & SynCAM2 & / \\
\hline $\mathrm{Necl}-4$ & TSLL2, SynCAM4 & Suppresseur de tumeur dans les gliomes et le cancer du poumon \\
\hline $\mathrm{Necl}-5$ & Tage4, PVR, CD155 & $\begin{array}{l}\text { Récepteur d'entrée pour le virus de la poliomyélite } \\
\text { Surexprimée dans les carcinomes }\end{array}$ \\
\hline
\end{tabular}

Tableau I. Nomenclature des nectines et des nectines-like. Implication en pathologie humaine et phénotype des souris knock-out. 


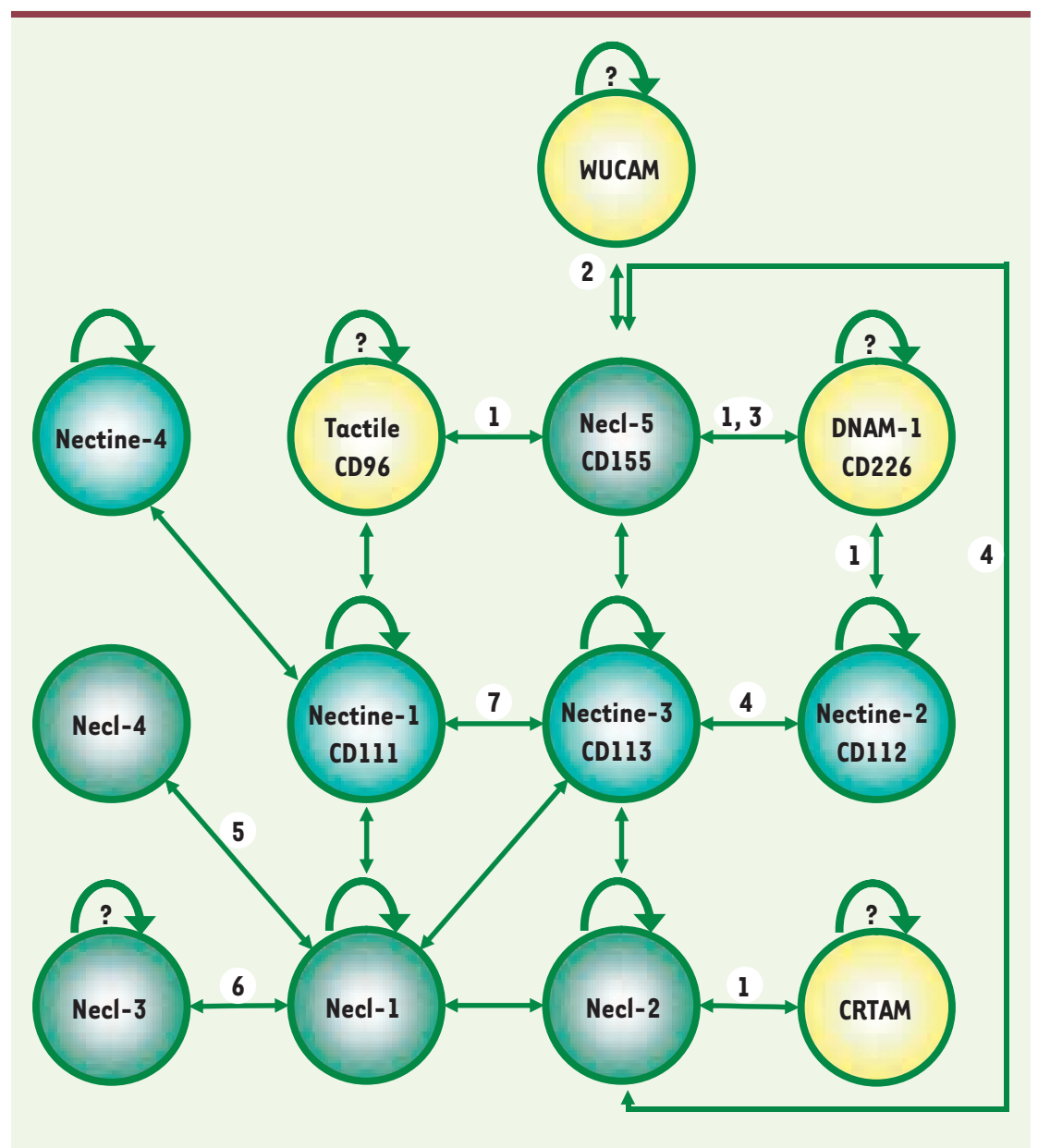

Figure 2. Trans-interactions des nectines et des nectines-like. Les interactions homophiliques (flèche arrondie) et hétérophiliques (flèche droite à double direction) ont été décrites à partir de tests biochimiques et cellulaires. Le «? » signifie qu'il n'existe pas de données concernant l'interaction homophilique. Certaines de ces interactions ont été décrites dans un contexte physiologique. 1. Surveillance immunitaire (interaction lymphocytes T cytolytiques/cellules tumorales). 2. Régulation du système immunitaire (interaction lymphocytes T CD4/cellules dendritiques folliculaires). 3. Diapédèse (interaction monocyte/cellule endothéliale). 4. Spermiogenèse (interaction cellules germinales/cellules de Sertoli). 5. Système nerveux (interaction axones/cellules de Schwann). 6. Système nerveux (interaction axones/oligodendrocytes). 7. Système nerveux (jonction pré- et post-synaptique).

des cellules tumorales par les cellules NK et les cellules T cytotoxiques. Il est important de noter que necl- 5 possède 4 ligands différents dont 3 sont impliqués dans la surveillance immunitaire (DNAM-1, tactile et WUCAM). L'engagement de tactile avec necl-5 augmente dans certaines circonstances la lyse NK [25] (Figure 3B).

Necl-5 est exprimée au niveau des jonctions interendothéliales et DNAM-1 au niveau des monocytes. L'interaction entre necl-5 et DNAM-1 contrôle la diapédèse ${ }^{2}$ trans-endothéliale des monocytes [26]. De la même façon, cette interaction pourrait aussi faciliter la diapédèse trans-endothéliale des cellules NK au site tumoral.

In vivo, il a aussi été démontré que la surexpression de necl-5 augmente la formation de métastases au niveau du poumon dans un modèle murin dans lequel les cellules tumorales sont injectées dans la veine caudale.

Dans d'autres expériences, la surexpression de nectine-2 et necl-5 augmente les propriétés prolifératives, migratoires et invasives des cellules [27, 28]. Necl-5

${ }^{2}$ Migration des monocytes entre les cellules endothéliales pour passer de la circulation dans le sous-endothélium. contribue à la régulation des phénomènes d'inhibition de contact en modulant la signalisation via l'intégrine alpha(v)-béta3. La transformation cellulaire induite par l'oncogène KRAS V12 induit une augmentation marquée de l'expression de necl-5 et la perte d'inhibition de contact [29].

Nectine-2 et surtout necl-5 représentent des acteurs importants en cancérologie car ils interviennent dans les étapes clés de la progression tumorale: prolifération, migration, et formation de métastases. Face à la surexpression de ces antigènes, le système immunitaire n'est pas capable de contrôler le développement tumoral chez l'homme.

\section{Nectine-4 : un marqueur des carcinomes mammaires et du poumon}

Les nectines et les nectines-like sont exprimées dans les tissus embryonnaires et adultes. Cependant, par rapport aux autres membres de la famille, nectine-4 présente un profil d'expression unique car normalement restreint au stade embryonnaire [4]. La protéine est réexprimée dans des tumeurs mammaires de mauvais pronostic alors qu'elle est absente du tissu épithélial mammaire sain [30]. Une forme soluble, correspondant à la région extracellulaire de nectine-4, est générée par clivage protéolytique impliquant la métalloprotéase ADAM17 ( $A$ disintegrin and metalloproteinase domain 17 encore appelée TACE pour tumor necrosis factor- $\alpha$-converting enzyme) [31, 41]. Cette forme soluble est détectée dans le sérum de patientes atteintes de carcinome 


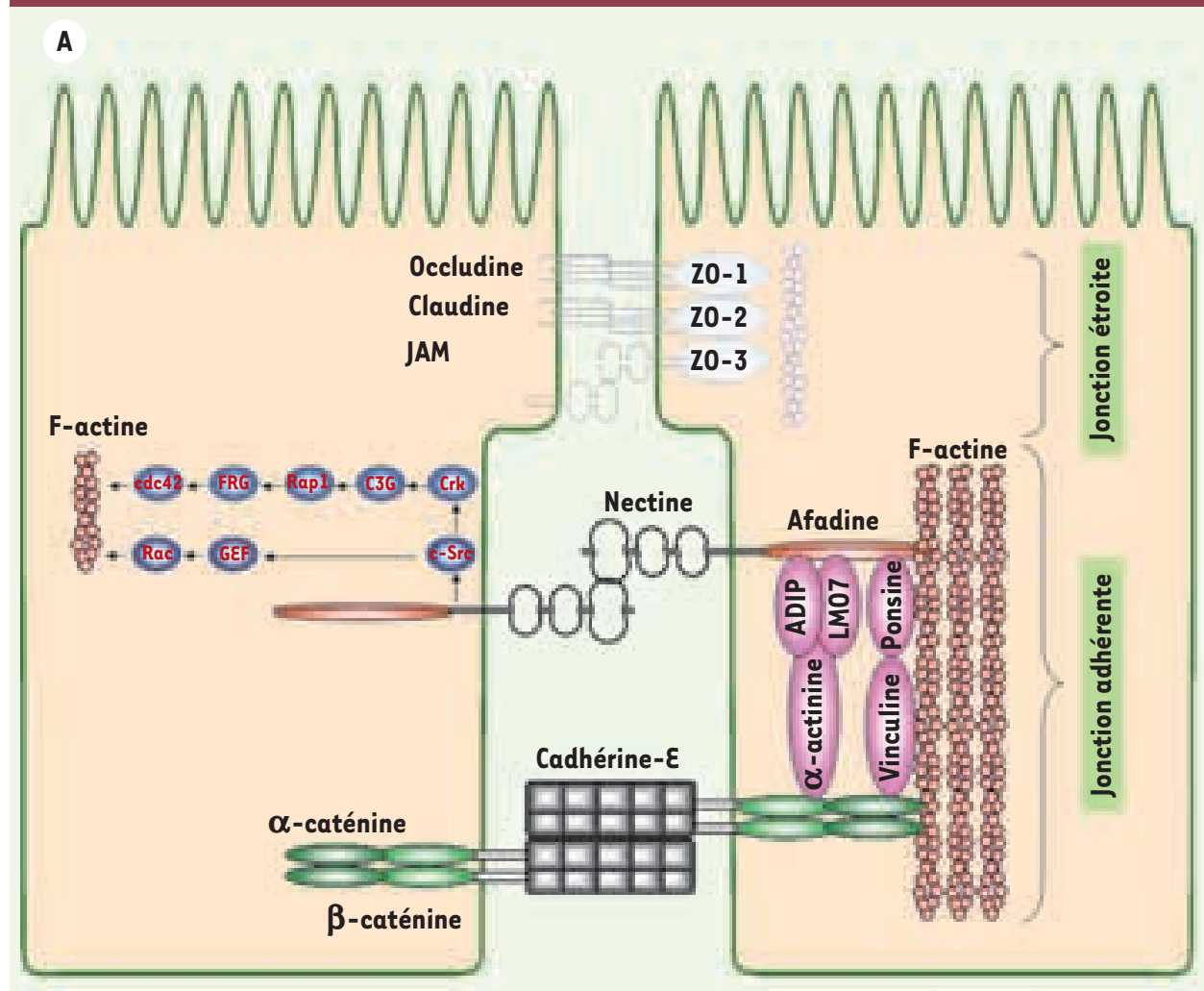

Figure 3. Rôle des nectines et nectines-like dans les interactions intercellulaires. A. Les nectines, constituants des jonctions adhérentes épithéliales. Partie droite: la localisation des nectines dans une cellule épithéliale polarisée. L'interaction des nectines avec l'afadine est déterminante dans ce contexte. Partie gauche: la signalisation intracellulaire des nectines aboutissant à l'activation de RAC-l et CDC42. Cette activation est nécessaire au cours de la formation des JA et dans la migration cellulaire. $B$. Les récepteurs NK interagissent avec les nectines et les nectines-like exprimées à la surface des cellules tumorales. L'engagement de ces récepteurs favorise l'adhérence hétérotypique et dans certains cas active les fonctions cytotoxiques des cellules NK.

dans le sérum de patients atteints de carcinomes pulmonaires non à petites cellules. Combinée à l'ACE ou au CYFRA21-1 (cytokeratin-21 fragment), nectine-4 sérique permet d'augmenter la sensibilité et la spécificité de la détection précoce de tumeurs [32].

mammaire métastatique et représente un nouveau marqueur sérique complémentaire des marqueurs classiquement utilisés comme l'ACE (antigène carcinoembryonnaire) et le CA-15.3. Nectine-4 est un antigène de type carcinoembryonnaire. Son absence d'expression dans les tissus adultes normaux en fait une cible candidate en immunothérapie. Récemment, nectine-4 a été identifiée

\section{Gènes suppresseurs de tumeurs}

\section{Necl-1 et necl-4 : gènes suppresseurs de tumeurs dans les gliomes et les tumeurs du côlon}

Necl-l a une expression limitée au tissu neural où elle joue un rôle dans la synaptogenèse et la myélinisation du système nerveux [33]. L'expression de necl-l est perdue dans des gliomes. Cette perte 
d'expression est le résultat de l'augmentation de l'activité des enzymes histone déacétylases. La perte d'expression de necl-l est impliquée dans la tumorigenèse. L'expression ectopique de necl-1 dans des cellules tumorales permet de diminuer la croissance tumorale de cellules injectées dans la souris nude. Elle réprime également la prolifération cellulaire en provoquant l'arrêt du cycle cellulaire [34] et suggère que necl-1 est un suppresseur de tumeur. Une étude récente montre que necl-1 et necl-4 réduisent la tumorigénicité des cellules tumorales de côlon greffées dans la souris nude [35].

\section{Necl-2 : un gène suppresseur de tumeur dans le cancer du poumon}

Necl-2 est fréquemment inactivée dans les carcinomes du poumon, du pancréas et du foie [36]. Cette inactivation est le résultat de la perte d'un allèle du gène et de la méthylation du promoteur de l'autre allèle. L'expression ectopique de necl-2 dans des cellules de carcinome du poumon supprime leur pouvoir tumorigène dans un modèle de xénogreffe chez la souris nude. De plus, necl-2 induit l'apoptose et l'arrêt de la prolifération cellulaire [37].

Necl-2 est un ligand du récepteur CRTAM (cytotoxic and regulatory $T$ cell molecule) exprimé par les cellules NK, les lymphocytes $\operatorname{CD}^{+}$et une souspopulation de lymphocytes CD4 ${ }^{+}$. CRTAM est un régulateur de la polarité lymphocytaire T et l'interaction entre necl-2 et CRTAM est responsable de l'activité cytotoxique des cellules NK et des lymphocytes CD8 $8^{+}$[38] (Figure 3B). Ainsi l'activité intrinsèque de gène suppresseur de tumeur de necl-2 explique que ses anomalies puissent participer au développement tumoral probablement par l'absence de contrôle du système immunitaire.

\section{Afadine/AF-6 : un marqueur des cancers du sein de mauvais pronostic}

L'afadine est le partenaire cytoplasmique privilégié des nectines. La région contenant le gène de l'afadine (MLLT4, myeloid/lymphoid or mixed-lineage leukemia, localisé en 6q27) est altérée dans les cancers de l'ovaire et du sein $[39,40]$. Dans les leucémies aiguës myéloïdes, MLLT4 est un partenaire de fusion de MLL. L'afadine/AF-6 est un potentiel gène suppresseur de tumeurs.

L'afadine/AF-6 est exprimée de façon ubiquitaire dans les cellules épithéliales mais est absente dans environ $15 \%$ des carcinomes mammaires. Cette perte d'expression est corrélée avec une cassure au niveau du site FRA6E (6q26). La perte d'afadine/AF-6 semble être un marqueur métastatique associé à un mauvais pronostic pour les patientes. Ce marqueur permettrait aussi de définir des patientes à risque, diagnostiquées initialement avec un bon pronostic [40].

L'afadine/AF-6 est impliquée dans la physiologie épithéliale. Cette protéine plateforme relie au cytosquelette d'actine des protéines d'adhérence, des récepteurs cellulaires et des effecteurs de voies de signalisation [16]. L'absence d'afadine/AF-6 contribuerait au développement tumoral et à la formation de métastases en perturbant la polarité épithéliale et l'adhérence cellulaire.

\section{Conclusion}

Les nectines et les nectines-like forment une famille de molécules d'adhérence impliquées dans des fonctions physiologiques très diverses. Ces fonctions peuvent être perturbées dans les pathologies, notamment dans les processus d'infection, de migration et de transmigration. Cette famille présente la particularité de partager des ligands de la SFIg impliqués d'une part dans l'adhérence cellulaire et d'autre part dans les fonctions cytotoxiques lymphocytaires T et NK. D'un point de vue clinique, ces molécules ont un intérêt en tant que marqueurs histologique (nectine-4, afadine/AF6) et sérique (nectine4), mais ce sont aussi de bons candidats cibles de thérapies innovantes: biothérapie (anticorps monoclonaux anti-necl5 , anti-nectine-4), vaccination thérapeutique et prophylactique (nectine-4), thérapie oncolytique utilisant le virus herpès (nectine-1). Le développement de modèles précliniques doit permettre de répondre à certains de ces points.

On connaît encore peu de choses sur les mécanismes de la régulation de l'expression de ces molécules et les mécanismes d'action de ces molécules dans la progression tumorale. Il est probable que les niveaux d'expression, l'interaction avec les ligands et la signalisation via les régions cytoplasmiques jouent un rôle central dans ces mécanismes. $\diamond$

\section{SUMMARY}

Nectin and nectin-like molecules as markers, actors and targets in cancer

Nectin and nectin-like (necl) proteins form a family of 9 adhesion molecules that belong to the immunoglobulin superfamily. They play a key role in different biological processes such as cell polarity, proliferation, differentiation and migration in epithelial, endothelial, immune and nervous systems. Besides their role in physiology, they have been involved in different pathological processes in humans. They serve as virus receptors (poliovirus and herpes simplex virus), they are involved in orofacial malformation (CLPEDI) and recently they have been described as markers, actors and potential therapeutics targets in cancer. Among them, necl-5, nectin-2 and nectin-4 are overexpressed in tumors, and are associated with a poor prognosis. On the opposite, necl-1, necl-2 and necl-4 act as tumor suppressors and are repressed in cancer. The involvement of nectins and necls molecules in cancer and their potential used in therapy is discussed in this review. $\diamond$

\section{CONFLIT D'INTÉRÊTS}

Les auteurs déclarent n'avoir aucun conflit d'intérêts concernant les données publiées dans cet article.

\section{REMERCIEMENTS}

Merci à Daniel Birnbaum, Patrice Dubreuil et Stéphanie Fabre-Lafay pour leurs précieux conseils. Le laboratoire d'oncologie moléculaire est soutenu par l'Inserm, l'Institut Paoli-Calmettes et la Ligue contre le cancer (équipe labellisée). 


\section{GLOSSAIRE}

ACE : antigène carcinoembryonnaire

ADAM17 : a disintegrin and metalloproteinase 17

ADIP : afadin DIL domain-interacting protein

CLPED1 : cleft lip palate ectodermal dysplasia 1

CRTAM : class I restricted T cell-associated molecule

CYFRA21-1: cytokeratin-21 fragment

DAL1 : differentially expressed in adenocarcinoma of the lung

DNAM1 : Dnax-accessory molecule 1

IQGAP1:I0 motif containing GTPase activating protein 1

MAGUK : membrane associated guanylate kinase MLLT4 : myeloid/lymphoid or mixed-lineage leukemia PAR3 : partitioning defective-3

TACE : tumor necrosis factor- $\alpha$-converting enzyme WUCAM : Washington University cell adhesion molecule

\section{RÉFÉRENCES}

1. Lopez M, Eberle F, Mattei MG, et al. Complementary DNA characterization and chromosomal localization of a human gene related to the poliovirus receptor-encoding gene. Gene 1995 ; 155 : 261-5.

2. Lopez M, Aoubala M, Jordier F, et al. The human poliovirus receptor related 2 protein is a new hematopoietic/endothelial homophilic adhesion molecule. Blood 1998; 92 : 4602-11.

3. Reymond N, Borg JP, Lecocq $\varepsilon$, et al. Human nectin3/PRR3: a novel member of the PVR/PRR/nectin family that interacts with afadin. Gene 2000 ; $255: 347-55$

4. Reymond N, Fabre S, Lecocq $\varepsilon$, et al. Nectin4/PRR4, a new afadinassociated member of the nectin family that trans-interacts with nectinl/ PRRl through V domain interaction. J Biol Chem 2001 ; 276 : 43205-15.

5. Fukuhara $H$, Kuramochi M, Nobukuni T, et al. Isolation of the TSLLI and TSLL2 genes, members of the tumor suppressor TSLCl gene family encoding transmembrane proteins. Oncogene 2001; 20 : 5401-7.

6. Urase K, Soyama A, Fujita $\varepsilon$, Momoi T. Expression of RA175 mRNA, a new member of the immunoglobulin superfamily, in developing mouse brain. Neuroreport $2001 ; 12$ : 3217-21.

7. Biederer T, Sara Y, Mozhayeva M, et al. SynCAM, a synaptic adhesion molecule that drives synapse assembly. Science 2002 ; 297 : 1525-31.

8. Mendelsohn CL, Wimmer $\varepsilon$, Racaniello VR. Cellular receptor for poliovirus: molecular cloning, nucleotide sequence, and expression of a new member of the immunoglobulin superfamily. Cell $1989 ; 56: 855-65$.

9. Takahashi K, Nakanishi H, Miyahara M, et al. Nectin/PRR: an immunoglobulin-like cell adhesion molecule recruited to cadherin-based adherens junctions through interaction with Afadin, a PDZ domaincontaining protein. J Cell Biol 1999; $145: 539-49$.

10. Lopez M, Cocchi F, Avitabile $\varepsilon$. Novel, soluble isoform of the herpes simplex virus (HSV) receptor nectinl (or PRRl-HlgR-HveC) modulates positively and negatively susceptibility to HSV infection. J Virol $2001 ; 75: 5684-91$.

11. Sakisaka T, Takai Y. Biology and pathology of nectins and nectin-like molecules. Curr Opin Cell Biol 2004 ; 16 : 513-21.

12. Bottino C, Castriconi R, Pende D, et al. Identification of PVR (CD155) and Nectin-2 (CD112) as cell surface ligands for the human DNAM-1 (CD226) activating molecule. J Exp Med 2003 ; 198 : 557-67.

13. Seth S, Maier MK, Qiu Q, et al. The murine pan T cell marker CD96 is an adhesion receptor for CD155 and nectin-1. Biochem Biophys Res Commun 2007 ; 364 : 959-65.

14. Galibert L, Diemer GS, Liu Z, et al. Nectin-like protein 2 defines a subset of T-cell zone dendritic cells and is a ligand for class-I-restricted T-cellassociated molecule. J Biol Chem 2005 ; 280 : 21955-64.
15. Boles KS, Vermi W, Facchetti F, et al. A novel molecular interaction for the adhesion of follicular CD4 T cells to follicular DC. Eur J Immunol 2009 ; $39: 695-703$.

16. Takai $Y$, Ikeda W, Ogita H, Rikitake $Y$. The immunoglobulin-like cell adhesion molecule nectin and its associated protein afadin. Annu Rev Cell Dev Biol 2008 ; 24 : 309-42.

17. Kakunaga S, Ikeda W, Itoh S, et al. Nectin-like molecule-1/TSLL1/SynCAM3: a neural tissuespecific immunoglobulin-like cell-cell adhesion molecule localizing at non-junctional contact sites of presynaptic nerve terminals, axons and glia cell processes. J Cell Sci 2005 ; 118 : 1267-77.

18. Mueller $S$, Cao X, Welker R, Wimmer $\varepsilon$. Interaction of the poliovirus receptor CD155 with the dynein light chain Tctex-l and its implication for poliovirus pathogenesis. J Biol Chem 2002 ; $277: 7897-904$.

19. Lopez M, Campadelli-Fiume G, Dubreuil P. Une nouvelle famille de molécules d'adhérence identifiée comme récepteur des virus herpes simplex. Med Sci (Paris) 1999 ; 15 : 1045-7.

20. Suzuki K, Hu D, Bustos T, et al. Mutations of PVRL1, encoding a cell-cell adhesion molecule/ herpesvirus receptor, in cleft lip/palate-ectodermal dysplasia. Nat Genet $2000 ; 25: 427-30$.

21. Castriconi R, Daga A, Dondero A, et al. NK cells recognize and kill human glioblastoma cells with stem cell-like properties. J Immunol 2009; 182 : 3530-9.

22. Carlsten M, Bjorkstrom NK, Norell $\mathrm{H}$, et al. DNAX accessory molecule-l mediated recognition of freshly isolated ovarian carcinoma by resting natural killer cells. Cancer Res 2007 ; 67 : 1317-25.

23. Tahara-Hanaoka S, Shibuya K, Kai H, et al. Tumor rejection by the poliovirus receptor family ligands of the DNAM-1 (CD226) receptor. Blood 2006; 107 : 1491-6.

24. Iguchi-Manaka A, Kai H, Yamashita $Y$, et al. Accelerated tumor growth in mice deficient in DNAM-1 receptor. J Exp Med 2008 ; $205: 2959-64$.

25. Fuchs A, Cella M, Giurisato $\varepsilon$, Shaw AS, Colonna M. Cutting edge: CD96 (tactile) promotes NK celltarget cell adhesion by interacting with the poliovirus receptor (CD155). J Immunol 2004; $172: 3994-8$.

26. Reymond N, Imbert AM, Devilard $\varepsilon$, et al. DNAM- 1 and PVR regulate monocyte migration through endothelial junctions. J Exp Med 2004 ; 199 : 1331-41.

27. Sloan KE, Eustace BK, Stewart JK, et al. CD155/PVR plays a key role in cell motility during tumor cell invasion and migration. BMC Cancer $2004 ; 4: 73$.

28. Sloan KE, Stewart JK, Treloar AF, Matthews RT, Jay DG. CD155/PVR enhances glioma cell dispersal by regulating adhesion signaling and focal adhesion dynamics. Cancer Res $2005 ; 65: 10930-7$.

29. Hirota T, Irie K, Okamoto R, Ikeda W, Takai Y. Transcriptional activation of the mouse Necl-5/ Tage4/PVR/CD155 gene by fibroblast growth factor or oncogenic Ras through the Raf-MEK-ERKAP-1 pathway. Oncogene $2005 ; 24: 2229-35$.

30. Fabre-Lafay S, Monville F, Garrido-Urbani S, et al. Nectin-4 is a new histological and serological tumor associated marker for breast cancer. BMC Cancer $2007 ; 7: 73$.

31. Fabre-Lafay S, Garrido-Urbani S, Reymond N, et al. Nectin-4, a new serological breast cancer marker, is a substrate for tumor necrosis factor-alpha-converting enzyme (TACE)/ADAM-17. J Biol Chem $2005 ; 280$ : 19543-50.

32. Takano A, Ishikawa N, Nishino R, et al. Identification of nectin-4 oncoprotein as a diagnostic and therapeutic target for lung cancer. Cancer Res $2009 ; 69: 6694-703$.

33. Spiegel I, Adamsky K, Eshed Y, et al. A central role for Necl4 (SynCAM4) in Schwann cell-axon interaction and myelination. Nat Neurosci $2007 ; 10: 861-9$.

34. Gao J, Chen T, Liu J, et al. Loss of NECLL, a novel tumor suppressor, can be restored in glioma by HDAC inhibitor-Trichostatin A through Spl binding site. Glia 2009; 57 : 989-99.

35. Raveh S, Gavert N, Spiegel I, Ben-Ze'ev A. The cell adhesion nectin-like molecules (Necl) 1 and 4 suppress the growth and tumorigenic ability of colon cancer cells. J Cell Biochem 2009; $108: 326-36$.

36. Kuramochi M, Fukuhara $H$, Nobukuni $T$, et al. TSLCl is a tumor-suppressor gene in human nonsmall-cell lung cancer. Nat Genet $2001 ; 27: 427-30$.

37. Mao X, Seidlitz $\varepsilon$, Truant R, Hitt M, Ghosh HP. Re-expression of TSLCl in a non-small-cell lung cancer cell line induces apoptosis and inhibits tumor growth. Oncogene $2004 ; 23: 5632-42$.

38. Boles KS, Barchet W, Diacovo T, Cella M, Colonna M. The tumor suppressor TSLCl/NECL-2 triggers NK-cell and CD8+ T-cell responses through the cell-surface receptor CRTAM. Blood 2005; $106: 779-86$

39. Minaguchi T, Matsushima M, Saito S, et al. Complete DNA sequence and characterization of a 330 $\mathrm{kb}$ VNTR-rich region on chromosome $6 \mathrm{q} 27$ that is commonly deleted in ovarian cancer. DNA Res $1999 ; 6: 131-6$

40. Letessier A, Garrido-Urbani S, Ginestier C, et al. Correlated break at PARK2/FRA6E and loss of AF-6/Afadin protein expression are associated with poor outcome in breast cancer. Oncogene $2007 ; 26: 298-307$.

41. Peiretti F, Canault M, Morange P, Alessi MC, Nalbone G. Les deux visages d'ADAM17 dans l'inflammation : implications dans l'athérosclérose et l'obésité. Med Sci (Paris) 2009 ; 25 : 45-50
TIRÉS À PART

G. Fournier et M. Lopez 

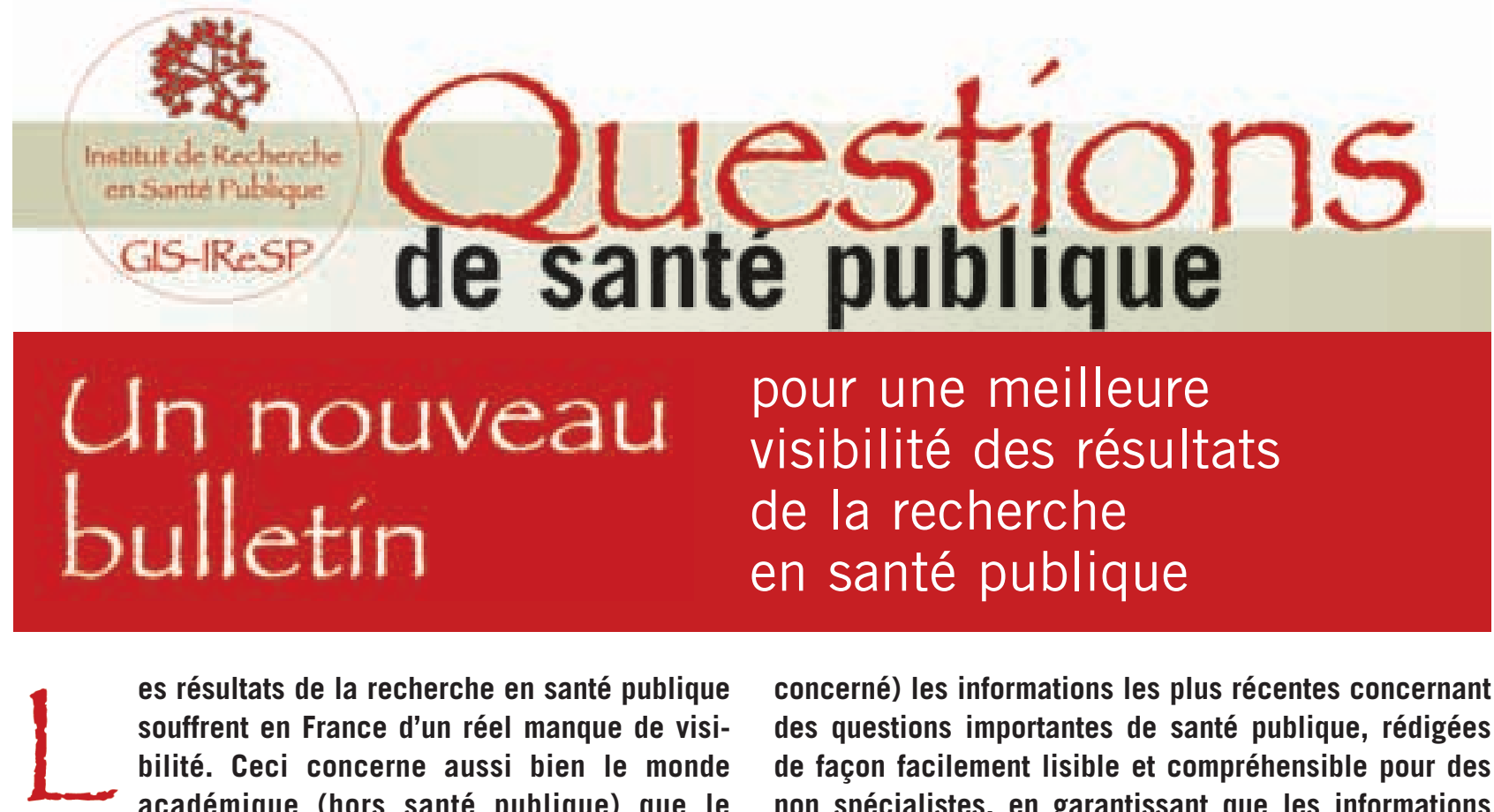

es résultats de la recherche en santé publique souffrent en France d'un réel manque de visibilité. Ceci concerne aussi bien le monde académique (hors santé publique) que le grand public et les décideurs. Pour pallier ce déficit, I'IReSP crée un bulletin à large diffusion intitulé "Questions de santé publique ", largement inspiré du bulletin mensuel d'information de l'INED « Populations et sociétés ". L'objectif éditorial est de porter à la connaissance d'un large public (enseignants, étudiants, journalistes, décideurs, milieux de la recherche, associations, public

concerné) les informations les plus récentes concernant des questions importantes de santé publique, rédigées de façon facilement lisible et compréhensible pour des non spécialistes, en garantissant que les informations publiées sont validées scientifiquement. La publications concernera des faits et non des positions. Au-delà de la présentation de résultats, cette publication devrait également avoir des qualités pédagogiques, permettant au lecteur de mieux comprendre comment sont formulées et abordées les questions de santé publique et quelles sont les limites de ces études.

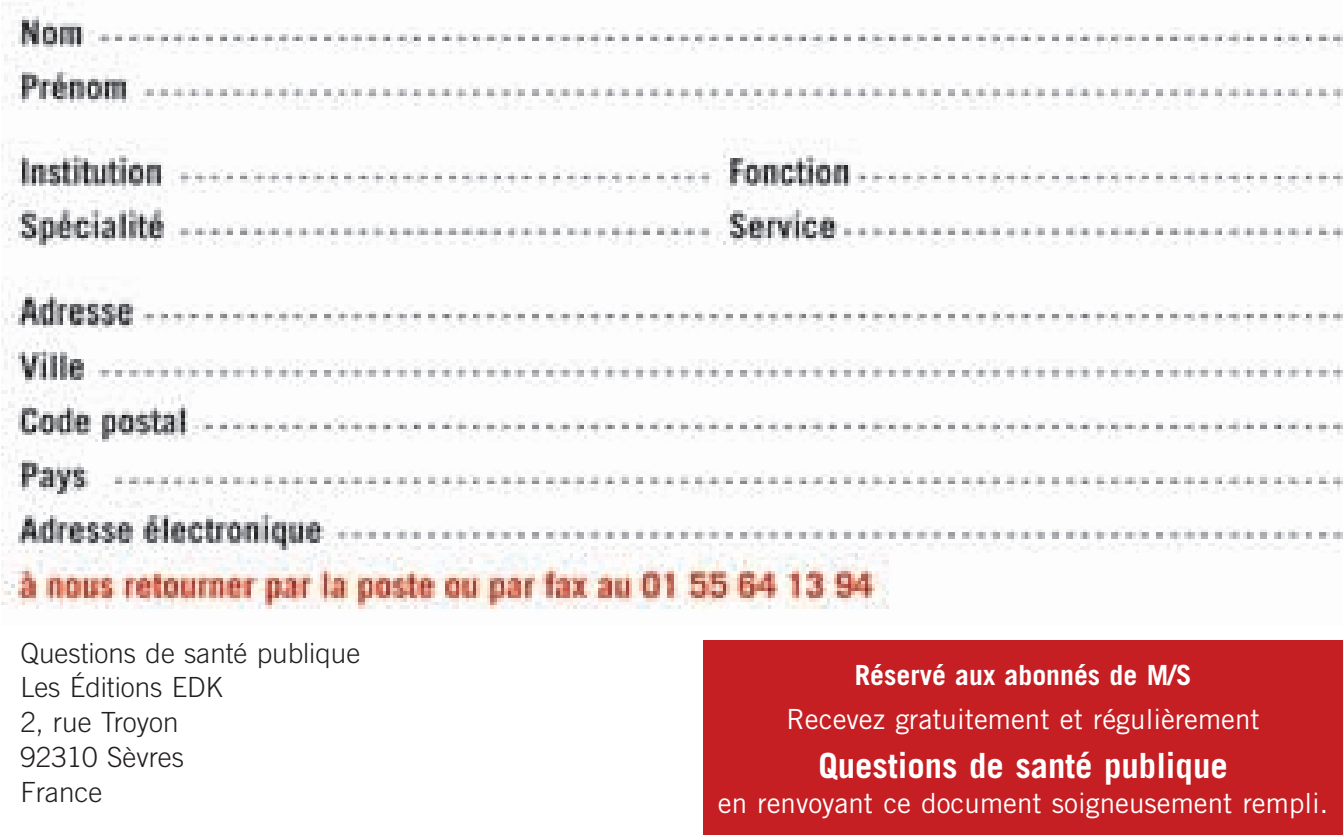

Questions de santé publique est une publication de l'Institut de Recherche en Santé Publique. I Directeur de la publication : Alfred Spira. I Rédacteur en chef : Nathalie de Parseval. I Une réalisation des Éditions EDK. 